Pacific Journal of Mathematics

SCALE-INVARIANT MEASURABILITY IN ABSTRACT WIENER 


\section{SCALE-INVARIANT MEASURABILITY IN ABSTRACT WIENER SPACES}

\section{Dong Myung Chung}

In this paper, we first prove a limit theorem for a sequence of quadratic functionals on an abstract Wiener space which generalizes a Cameron-Martin limit theorem in the Wiener space; and next we prove a version of a converse measurability theorem for the Wiener space in the setting of abstract Wiener spaces. Using these results, we discuss scaleinvariant measurability and translations in an abstract Wiener space.

1. Introduction and preliminaries. Let $H$ be a real separable infinite dimensional Hilbert space with inner product $\langle\cdot, \cdot\rangle$ and norm $|\cdot|$. Let $\mu$ be the cylinder set measure on $H$ defined by

$$
\mu(A)=(2 \pi)^{-n / 2} \int_{F} \exp \left\{-\frac{|x|^{2}}{2}\right\} d x,
$$

where $A=P^{-1}(F), F$ is a Borel set in the image of an $n$-dimensional projection $P$ in $H$ and $d x$ is Lebesgue measure in $P H$. A norm $\|\cdot\|$ on $H$ is called measurable if for every $\varepsilon>0$ there exists a finite dimensional projection $P_{0}$ such that $\mu(\{x \in H:\|P x\|>\varepsilon\})<\varepsilon$ whenever $P$ is a finite dimensional projection orthogonal to $P_{0}$. It is known (see [8]) that $H$ is not complete with respect to $\|\cdot\|$. Let $B$ denote the completion of $H$ with respect to $\|\cdot\|$. Let $i$ denote the natural injection from $H$ into $B$. The adjoint operator $i^{*}$ is one-to-one and maps $B^{*}$ continuously onto a dense subset of $H^{*}$. By identifying $H^{*}$ with $H$ and $B^{*}$ with $i^{*} B^{*}$, we have a triple $B^{*} \subset H \subset B$ and $\langle x, y\rangle=(x, y)$ for all $x$ in $H$ and $y$ in $B^{*}$, where $(\cdot, \cdot)$ denote the natural dual pairing between $B$ and $B^{*}$. By a well-known result of Gross, $\mu \circ i^{-1}$ has a unique countably additive extension $\nu$ to the Borel $\sigma$-algebra $\mathscr{B}(B)$ of $B$. The triple $(H, B, \nu)$ is called an abstract Wiener space. For more details, see Kuo [8].

Let $C_{1}[a, b]$ denote the Banach space $\{x(\cdot): x$ is a continuous function with $x(a)=0\}$ with the uniform norm. Let $\left(C_{1}[a, b]\right.$, $\left.\mathscr{B}\left(C_{1}[a, b]\right), m_{w}\right)$ denote the Wiener space, where $m_{w}$ is the Wiener measure on the Borel $\sigma$-algebra $\mathscr{B}\left(C_{1}[a, b]\right)$ of $C_{1}[a, b]$ (see [12]), and let $C_{1}^{\prime}[a, b]=\left\{x \in C_{1}[a, b]: x(t)=\int_{a}^{t} f(u) d u, f \in L^{2}[a, b]\right\}$; then it is a real separable infinite dimensional Hilbert space with the inner product $\left\langle x_{1}, x_{2}\right\rangle=\int_{a}^{b} D x_{1}(t) \cdot D x_{2}(t) d t$, where $D x=d x / d t$. 
Let $Q=(s, t): a \leq s \leq b$ and $c \leq t \leq d$ and let $C_{2}[Q]$ denote the Banach space $\{x(\cdot, \cdot): x$ is a continuous function on $Q$ with $x(a, t)=$ $x(s, c)=0\}$ with the uniform norm. Let $\left(C_{2}[Q], \mathscr{B}\left(C_{2}[Q]\right), m_{y}\right)$ denote the Yeh-Wiener space [13], i.e. $m_{y}$ is the probability measure on the Borel $\sigma$-algebra $\mathscr{B}\left(C_{2}[Q]\right)$ of $C_{2}[Q]$ such that

$$
\begin{array}{r}
m_{y}(W)=\prod_{j=1}^{m} \prod_{k=1}^{n}\left\{2 \pi\left(s_{j}-s_{j-1}\right)\left(t_{k}-t_{k-1}\right)\right\}^{-1 / 2} \\
\int_{p_{m, n}}^{q_{m, n}} \cdots \int_{p_{1,1}}^{q_{1,1}} \exp \left\{-\sum_{j=1}^{m} \sum_{k=1}^{n} \frac{\left[u_{j, k}-u_{j-1, k}-u_{j, k-1}+u_{j-1, k-1}\right]^{2}}{2\left(s_{j}-s_{j-1}\right)\left(t_{k}-t_{k-1}\right)}\right\} \\
\times d u_{11} \cdots d u_{m n} .
\end{array}
$$

where $W=\left\{x \in C_{2}[Q]: p_{j, k} \leq x\left(s_{j}, t_{k}\right) \leq q_{j, k}\right.$, for $j=1, \ldots, m$ and $k$ $=1, \ldots, n\}$ and $u_{0, k}=u_{j, 0}=u_{0,0}=0$ for all $j$ and $k$. Let $C_{2}^{\prime}[Q]=\{x \in$ $\left.C_{2}[Q]: x(s, t)=\int_{a}^{s} \int_{c}^{t} f(u, v) d u d v, f \in L^{2}[Q]\right\}$; then it is a real separable infinite dimensional Hilbert space with the inner product

$$
\left\langle x_{1}, x_{2}\right\rangle=\int_{a}^{b} \int_{c}^{d} D^{2} x_{1}(s, t) \cdot D^{2} x_{2}(s, t) d s d t
$$

where $D^{2} x=\partial^{2} x / \partial t \partial s$. It is known [9] that the uniform norms on $C_{1}^{\prime}[a, b]$ and $C_{2}^{\prime}[Q]$ are measurable and that $\left(C_{1}^{\prime}[a, b], C_{1}[a, b], m_{w}\right)$ and $\left(C_{2}^{\prime}[Q], C_{2}[Q], m_{y}\right)$ are both examples of abstract Wiener spaces.

Let $\left\{e_{j}, j \geq 1\right\}$ be a complete orthonormal system in $H$ such that the $e_{j}$ 's are in $B^{*}$. For each $h$ in $H$ and $x$ in $B$, let

$$
(h, x)^{\sim}= \begin{cases}\lim _{n \rightarrow \infty} \sum_{j=1}^{n}\left\langle h, e_{j}\right\rangle\left(x, e_{j}\right), & \text { if the limit exists } \\ 0, & \text { otherwise. }\end{cases}
$$

Then $(h, \cdot)^{\sim}$ is a Borel measurable functional on $B$ and if both $h$ and $x$ are in $H$, Parseval's identity gives $(h, x)^{-}=\langle h, x\rangle$. (See [7].)

Let $\left\{\phi_{j}: j \geq 1\right\}$ be a sequence of functions of bounded variation on $Q$ which forms a complete orthonormal system of $L^{2}[Q]$. Let $v$ be in $L^{2}[Q]$ and

$$
v_{n}(s, t)=\sum_{j=1}^{n} \phi_{j}(s, t) \int_{a}^{b} \int_{c}^{d} v(s, t) \cdot \phi_{j}(s, t) d s d t .
$$

The Paley-Wiener-Zygmund integral of $v,(\phi) \int_{a}^{b} \int_{c}^{d} v(s, t) \tilde{d} x(s, t)$, is defined as

$$
\begin{aligned}
(\phi) \int_{a}^{b} \int_{c}^{d} v(s, t) \tilde{d x}(s, t) & \\
\quad & \begin{cases}\lim _{n \rightarrow \infty} \int_{a}^{b} \int_{c}^{d} v_{n}(s, t) d x(s, t), & \text { if the limit exists } \\
0, & \text { otherwise. }\end{cases}
\end{aligned}
$$


Motivated by the well-known fact that change of scale is a pathological transformation in Wiener space, Johnson and Skoug [6] introduced the concept of scale-invariant measurability in Wiener space and examined the exact nature of this concept, and then they applied their results to clarify conceptual subtleties in the theory of the Feynman integral and in the theory of the Fourier-Feynman transform. Later, scale-invariant measurability in Yeh-Wiener space was studied by Chang [3]. In the works of [6] and [3], one notes that the keys to their discussion are a limit theorem in Wiener space due to Cameron-Martin [2] and in Yeh-Wiener space due to Skoug [11], and a converse measurability theorem for Wiener space due to Koehler [10] and for Yeh-Wiener space due to Skoug [10]. In view of this, for the extension to the abstract Wiener space setting of the results obtained in $[6,3]$, the question is to look for suitable versions of those two results in the setting of abstract Wiener spaces.

In $\$ 2$, we prove a limit theorem for a sequence of quadratic functionals on an abstract Wiener space $(H, B, \nu)$ which is a generalization of a limit theorem in Wiener space [2] and in Yeh-Wiener space [11]. In §3, we prove a version of converse measurability theorem for Wiener space [10] and Yeh-Wiener space [11] in the setting of abstract Wiener spaces. In $\$ 4$, we extend the results on scale-invariant measurability and translations obtained in $[3,6]$ to an abstract Wiener space.

2. A limit theorem in abstract Wiener space. In this section, we prove a limit theorem for a sequence of quadratic functionals defined on an abstract Wiener space $(H, B, \nu)$ and then we exhibit a subset of $B$ with $\nu$-measure one which for all real $\alpha \neq \pm 1$ is transformed into a set of $\nu$-measure zero by the change of scale transformations $x \rightarrow \alpha x$. These are generalizations to the abstract Wiener space setting of the results first obtained by Levy [Amer. J. Math. 62 (1940), 487-550] and independently, but later, by Cameron and Martin [2] in Wiener space and extended by Skoug [11] to Yeh-Wiener space. We now begin with the following facts taken from [7].

LEMMA 2.1. Let $(H, B, \nu)$ be an abstract Wiener space.

(i) For each $h(\neq 0)$ in $H$, the random variable $x \rightarrow(h, x)^{\sim}$ is Gaussian with mean zero and variance $|h|^{2}$.

(ii) $(h, \alpha x)^{\sim}=\alpha(h, x)^{\sim}$ for any real number $\alpha, h \in H$ and $x \in B$.

(iii) If $\left\{h_{1}, h_{2}, \ldots, h_{n}\right\}$ is an orthonormal set in $H$, then the random variables $\left(h_{i}, x\right)^{\sim}$ 's are independent.

(iv) If $B=C_{2}[Q], H=C_{2}^{\prime}[Q]$, we have

$$
(h, x)^{\sim}=(\phi) \int_{Q} D^{2} h(s, t) \tilde{d x}(s, t) .
$$


TheOREM 2.2. Let $(H, B, \nu)$ be an abstract Wiener space. For each $n$, let $\left\{e_{1}^{n}, e_{2}^{n}, \ldots, e_{d(n)}^{n}\right\}$ be an orthonormal set in $H$ and let

$$
F_{n}(x)=\frac{1}{d(n)} \sum_{j=1}^{d(n)}\left[\left(e_{j}^{n}, x\right)^{\sim}\right]^{2}, \quad x \in B .
$$

(i) If $d(n) \geq n^{p}(p>0)$ for every $n$, then $F_{n}$ converges to 1 in $L^{2}(B, \nu)$ and hence in measure $\nu$.

(ii) If $d(n) \geq n^{p}(p>1)$ for every $n$, then $F_{n}$ converges to $1 \nu$-almost surely.

Proof (i) Since the random variables $\left(e_{j}^{n}, \cdot\right)^{\sim}$ 's are independent and Guassian with mean zero and variance one, it is easy to show that the mean of $F_{n}$ is one and the variance of $F_{n}, \operatorname{Var}\left(F_{n}\right)$, is $2 / d(n)$. Hence we have

$$
\int_{B}\left|F_{n}(x)-1\right|^{2} d \nu(x)=\frac{2}{d(n)} \leq \frac{2}{n^{p}} .
$$

This implies that $F_{n}$ converges to 1 in $L^{2}(B, \nu)$ and hence in measure $\nu$.

(ii) For each $n=2,3, \ldots$, let

$$
E_{n}=\left\{x \in B:\left|F_{n}(x)-1\right| \geq \frac{\log n}{\sqrt{n^{p-1}}}\right\} .
$$

Then, by Chebyshev's inequality [12], we have

$$
\nu\left(E_{n}\right) \leq \frac{n^{p-1}}{\log ^{2} n} \cdot \frac{2}{d(n)}<\frac{2}{n \cdot \log ^{2} n} .
$$

Since

$$
\sum_{\nu=2}^{\infty} \nu\left(E_{n}\right) \leq \sum_{n=2}^{\infty} \frac{2}{n \cdot \log ^{2} n}<\infty
$$

it follows from Borel-Cantelli's Lemma [12] that $\nu(F)=0$, where $F=$ $\lim \sup E_{n}$. Since $\lim _{n \rightarrow \infty}(\log n) / \sqrt{n^{p-1}}=0, F_{n}(x)$ converges to $1 \nu$-almost surely.

REMARK. It is worth pointing out that in Theorem 2.2, it is not important that $\left(e_{j}^{n}, \cdot\right)^{\sim}$ be Gaussian; in fact one can replace $\left(e_{j}^{n}, \cdot\right)^{\sim}$ 's by any triangular sequence $X_{1}^{n}, X_{2}^{n}, \ldots, X_{d(n)}^{n}$ of independent, identically distributed random variables on a probability space with mean zero and variance 1 . 
As an application of Theorem 2.2, we give the following example which shows how to obtain the corresponding result of Skoug's for Yeh-Wiener space [11].

EXAMPLe 2.3. Let $B=C_{2}[Q], H=C_{2}^{\prime}[Q]$ and $\nu=m_{y}$. For each $n$, let $a=s_{0}^{n}<s_{1}^{n}<\cdots<s_{g(n)}^{n}=b$ and $c=t_{0}^{n}<t_{1}^{n}<\cdots<t_{h(n)}^{n}=d$ be partitions of $[a, b]$ and $[c, d]$ respectively, and let

$$
\phi_{j, k}^{n}(s, t)=\frac{1}{\sqrt{\left(s_{j}^{n}-s_{j-1}^{n}\right)\left(t_{k}^{n}-t_{k-1}^{n}\right)}} \int_{a}^{s} \int_{c}^{t} I_{\left[s_{j-1}^{n}, s_{j}^{n}\right] \times\left[t_{k-1}^{n}, t_{k}^{n}\right]}(u, v) d u d v
$$

for $j=1,2, \ldots, g(n)$ and $k=1,2, \ldots, h(n)$. Then it is easily shown that for each $n,\left\{\phi_{j, k}^{n}\right\}$ is an orthonormal set in $C_{2}^{\prime}[Q]$, and for each $x \in C_{2}[Q]$, we have

$$
\begin{aligned}
\left(\phi_{j, k}^{n}, x\right)^{-} & =(\phi) \int_{a}^{b} \int_{c}^{d} D^{2} \phi_{j, k}^{n}(s, t) d x(s, t) \\
& =\frac{x\left(s_{j}^{n}, t_{k}^{n}\right)-x\left(s_{j-1}^{n}, t_{k}^{n}\right)-x\left(s_{j}^{n}, t_{k-1}^{n}\right)+x\left(s_{j-1}^{n}, t_{k-1}^{n}\right)}{\sqrt{\left(s_{j}^{n}-s_{j-1}^{n}\right)\left(t_{k}^{n}-t_{k-1}^{n}\right)}} .
\end{aligned}
$$

Let $p>1, g(n) \geq n^{p / 2}$ and $h(n) \geq n^{p / 2}$ for every $n$, and let $d(n)=$ $g(n) \cdot h(n)$. Then $d(n) \geq n^{p}$ for every $n$, and hence by Theorem 2.2,

$$
\begin{aligned}
& F_{n}(x)=\frac{1}{d(n)} \sum_{j=1}^{g(n)} \sum_{k=1}^{h(n)}\left[\left(\phi_{j, k}^{n}, x\right)^{\sim}\right]^{2} \\
& =\frac{1}{d(n)} \sum_{j=1}^{g(n)} \sum_{k=1}^{h(n)} \frac{\left[x\left(s_{j}^{n}, t_{k}^{n}\right)-x\left(s_{j-1}^{n}, t_{k}^{n}\right)-x\left(s_{j}^{n}, t_{k-1}^{n}\right)+x\left(s_{j-1}^{n}, t_{k-1}^{n}\right)\right]^{2}}{\left(s_{j}^{n}-s_{j-1}^{n}\right)\left(t_{k}^{n}-t_{k-1}^{n}\right)}
\end{aligned}
$$

converges to $1 \nu$-almost surely. In particular, if we take partitions of $[a, b]$ and $[c, d]$ such that

$$
s_{j}^{n}-s_{j-1}^{n}=\frac{b-a}{g(n)} \text { and } t_{k}^{n}-t_{k-1}^{n}=\frac{d-c}{h(n)},
$$

respectively, for all $j=1,2, \ldots, g(n)$ and $k=1,2, \ldots, h(n)$, then we have

$$
\begin{aligned}
F_{n}(x)=\frac{1}{(b-a)(d-c)} \sum_{j=1}^{g(n)} \sum_{k=1}^{h(n)} & {\left[x\left(s_{j}^{n}, t_{k}^{n}\right)-x\left(s_{j-1}^{n}, t_{k}^{n}\right)\right.} \\
& \left.-x\left(s_{j}^{n}, t_{k-1}^{n}\right)+x\left(s_{j-1}^{n}, t_{k-1}^{n}\right)\right]^{2}
\end{aligned}
$$


and hence

$$
\begin{gathered}
\lim _{n \rightarrow \infty} \sum_{j=1}^{g(n)} \sum_{k=1}^{h(n)}\left[x\left(s_{j}^{n}, t_{k}^{n}\right)-x\left(s_{j-1}^{n}, t_{k}^{n}\right)-x\left(s_{j}^{n}, t_{k-1}^{n}\right)+x\left(s_{j-1}^{n}, t_{k-1}^{n}\right)\right]^{2} \\
=(b-a)(d-c)
\end{gathered}
$$

which implies a result of Skoug in [11].

The following notation will be fixed throughout this paper: For each $\alpha>0$, let

$$
\begin{aligned}
& C_{\alpha}=\left\{x \in B: \lim _{n \rightarrow \infty} F_{n}(x)=\alpha^{2}\right\}, \\
& D=\left\{x \in B: \lim _{n \rightarrow \infty} F_{n}(x) \text { does not exist }\right\},
\end{aligned}
$$

where $\left\{F_{n}\right\}$ is the same as in Theorem 2.2 with $d(n) \geq n^{p}(p>1)$ for all $n$. Then it follows from Lemma 2.1 that $\beta C_{\alpha}=C_{\alpha \beta}$ for all $\beta>0$ and $\alpha \geq 0$. Clearly $C_{\alpha}(\alpha \geq 0)$ and $D$ are all Borel sets in $B$, and $B$ is the disjoint union of this family of sets.

The following results are generalizations of the results in [2] for Wiener space and in [11] for Yeh-Wiener space. The proof is an easy consequence of Theorem 2.2.

THeOREM 2.4. Let $(H, B, \nu)$ be an abstract Wiener space. Then

(i) $\nu\left(C_{\alpha}\right)=0$ if and only if $\alpha \neq 1$.

(ii) $\nu\left(\alpha^{-1} C_{\alpha}\right)=1$ for every $\alpha>0$.

(iii) $\nu\left(\beta^{-1} C_{\alpha}\right)=0$ for all $\alpha, \beta$ with $\alpha \neq \beta, \alpha \geq 0, \beta>0$.

(iv) $\nu(D)=0$.

3. Converse measurability theorem for abstract Wiener spaces. Let $a=s_{0}<s_{1}<\cdots<s_{m}=b$ and $c=t_{0}<t_{1} \cdots<t_{n}=d$ be partitions of $[a, b]$ and $[c, d]$ respectively. Let $F$ be any subset of Euclidean space $R^{m n}$ and let

$$
W=\left\{x \in C_{2}[Q]:\left(x\left(s_{1}, t_{1}\right), \ldots, x\left(s_{m}, t_{n}\right)\right) \in F\right\} .
$$

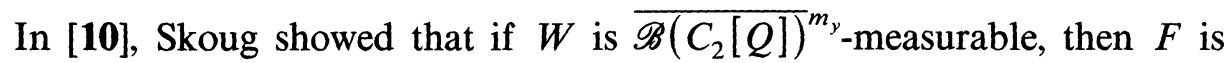
Lebesgue measurable in $R^{m n}$, here and in the rest of the paper, $\overline{\mathscr{F}}^{\lambda}$ denotes the completion of $\mathscr{F}$ with respect to a measure $\lambda$ on a measurable space $(X, \mathscr{F})$. In this section, we prove a version of this converse measurability theorem in the setting of abstract Wiener spaces. 
LEMMA 3.1. Let $X_{i}(i=1,2)$ be separable complete metric spaces and $\mathscr{B}\left(X_{i}\right)$ the o-algebra of Borel sets in $X_{i}$. Let $f: X_{1} \rightarrow X_{2}$ be a Borel measurable (i.e. $\mathscr{B}\left(X_{1}\right)-\mathscr{B}\left(X_{2}\right)$ measurable) function and let $\mu_{1}$ be a finite Borel measure on $X_{1}$ and $\mu_{2}=\mu_{1}^{\circ} f^{-1}$. If $A$ is a subset of $X_{2}$ such that $f^{-1}(A)$ is in $\overline{\mathscr{B}\left(X_{1}\right)^{\mu_{1}}}$, then $A$ is in $\overline{\mathscr{B}\left(X_{2}\right)^{\mu_{2}}}$.

Proof Since $f^{-1}(A)$ is in $\overline{\mathscr{B}\left(X_{1}\right)^{\mu_{1}}}$, there exists a Borel subset $B_{1}$ of $f^{-1}(A)$ such that $\mu_{1}\left(B_{1}\right)=\mu_{1}\left(f^{-1}(A)\right)$. Let $\varepsilon>0$ be given. Since $f$ is Borel measurable, it follows from a generalization of Lusin's Theorem [4, p. 227] that there exists a compact subset $K_{\varepsilon}$ of $B_{1}$ such that $\mu_{1}\left(B_{1}\right)-$ $\varepsilon / 2<\mu_{1}\left(K_{\varepsilon}\right)$ and $f$ restricted to $K_{\varepsilon}$ is continuous. Now let $f\left(K_{\varepsilon}\right)=E_{\varepsilon}$. Then $E_{\varepsilon}$ is a compact set in $X_{2}$ and $E_{\varepsilon} \subset A$, and we have

$$
\mu_{2}\left(E_{\varepsilon}\right)=\mu_{2}\left(f\left(K_{\varepsilon}\right)\right)=\mu_{1}\left(f^{-1}\left(f\left(K_{\varepsilon}\right)\right)\right) \geq \mu_{1}\left(K_{\varepsilon}\right) \geq \mu_{1}\left(B_{1}\right)-\varepsilon / 2 .
$$

Similarly, since $f^{-1}\left(A^{c}\right)$ is in $\overline{\mathscr{B}\left(X_{1}\right)^{\mu_{1}}}$, we can find a Borel subset $B_{2}$ of $f^{-1}\left(A^{c}\right)$ and a compact subset $L_{\varepsilon}$ of $A^{c}$ such that $\mu_{1}\left(B_{2}\right)=\mu_{1}\left(f^{-1}\left(A^{c}\right)\right)$ and $\mu_{2}\left(L_{\varepsilon}\right) \geq \mu_{1}\left(B_{2}\right)-\varepsilon / 2$. Let $L_{\varepsilon}^{c}=G_{\varepsilon}$. Then $E_{\varepsilon}$ and $G_{\varepsilon}$ are in $\mathscr{B}\left(X_{2}\right)$ and $E_{\varepsilon} \subset A \subset G_{\varepsilon}$, and we have

$$
\begin{aligned}
\mu_{2}\left(G_{\varepsilon}-E_{\varepsilon}\right) & =\mu_{2}\left(G_{\varepsilon}\right)-\mu_{2}\left(E_{\varepsilon}\right)=\mu_{2}\left(X_{2}\right)-\mu_{2}\left(L_{\varepsilon}\right)-\mu_{2}\left(E_{\varepsilon}\right) \\
& \leq \mu_{1}\left(X_{1}\right)-\left(\mu_{1}\left(B_{2}\right)-\varepsilon / 2\right)-\left(\mu_{1}\left(B_{1}\right)-\varepsilon / 2\right)=\varepsilon .
\end{aligned}
$$

Now letting $\varepsilon=1 / n, n=1,2, \ldots$, we obtain sequences $\left\{E_{n}\right\}$ and $\left\{G_{n}\right\}$ in $\mathscr{B}\left(X_{2}\right)$ such that $\mu_{2}\left(G_{n}-E_{n}\right) \leq 1 / n$. Let $F_{1}=\bigcap_{n=1}^{\infty} G_{n}$ and $F_{2}=$ $\bigcup_{n=1}^{\infty} E_{n}$. Then $F_{1}$ and $F_{2}$ are in $\mathscr{B}\left(X_{2}\right), F_{2} \subset A \subset F_{1}$, and $\mu_{2}\left(F_{1}-F_{2}\right)$ $=0$. Hence $A$ is in $\overline{\mathscr{B}\left(X_{2}\right)^{\mu_{2}}}$, completing of the proof of the lemma.

REMARK. It can be shown that Lemma 3.1 is still true with the same proof, for any Hausdorff topological space $X_{1}$ with finite Radon measure $\mu_{1}$ and any separable metric space $X_{2}$.

TheOREM 3.2. Let $(H, B, \nu)$ be an abstract Wiener space, and let $\left\{h_{1}, h_{2}, \ldots, h_{n}\right\}$ be a linearly independent subset of $H$. For any subset $F$ of Euclidean space $R^{n}$, let

$$
W=\left\{x \in B:\left(\left(h_{1}, x\right)^{\sim},\left(h_{2}, x\right)^{\sim}, \ldots,\left(h_{n}, x\right)^{\sim}\right) \in F\right\} .
$$

Then (i) $W$ is $\mathscr{B}(B)$-measurable if and only if $F$ is Borel measurable in $R^{n}$. (ii) $W$ is $\overline{\mathscr{B}(B)}{ }^{v}$-measurable if and only if $F$ is Lebesgue measurable in $R^{n}$.

Proof (i) Let $f(x)=\left(\left(h_{1}, x\right)^{\sim},\left(h_{2}, x\right)^{\sim}, \ldots,\left(h_{n}, x\right)^{\sim}\right), x \in B$. Then by Lemma $2.1, f$ is an $n$-dimensional Gaussian random vector on $B$ with 
mean zero and covariance $V=\left(v_{i j}\right)$ where $v_{i j}=\left\langle h_{i}, h_{j}\right\rangle$ for $i, j=$ $1,2, \ldots, n$. Hence if $F$ is Borel measurable in $R^{n}$, then $W=f^{-1}(F)$ is $\mathscr{B}(B)$-measurable. Conversely, suppose that $W$ is $\mathscr{B}(B)$-measurable. Since $\left\{h_{1}, h_{2}, \ldots, h_{n}\right\}$ is linearly independent in $H$, the matrix $V$ is non-singular. Let's define a function $g: R^{n} \rightarrow B$ by $g(u)=\sum_{j=1}^{n} v_{j} h_{j}$ where $\left(v_{1}, v_{2}, \ldots, v_{n}\right)=\left(u_{1}, u_{2}, \ldots, u_{n}\right) \cdot V^{-1}$ for $u=\left(u_{1}, u_{2}, \ldots, u_{n}\right) \in R^{n}$. Then it is easy to see that $G$ is a continuous (hence Borel measurable) function, and further we have $\left(\sum_{i=1}^{n} v_{i} h_{i}, h_{j}\right)^{\sim}=\left\langle\sum_{i=1}^{n} v_{i} h_{i}, h_{j}\right\rangle=u_{j}$ for every $j=1,2, \ldots, n$. Hence $u=\left(u_{1}, u_{2}, \ldots, u_{n}\right) \in F$ if and only if $G(u)$ $\in W$, equivalently $I_{F}(u)=I_{W} \circ G(u)$ for all $u \in R^{n}$. Therefore $F$ is $\mathscr{B}\left(R^{n}\right)$-measurable since $W$ is $\mathscr{B}(B)$-measurable. This completes the proof of (i).

(ii) Suppose that $F$ is a Lebesgue measurable set in $R^{n}$. Then there exist a Borel set $G$ and a subset $N_{1}$ of a Borel null set $N$ in $R^{n}$. Let $f$ be as in the proof of (i). Then since $f$ is Borel measurable, it follows that $f^{-1}(G)$ and $f^{-1}(N)$ are in $\mathscr{B}(B)$. Since $\nu \circ f^{-1}(N)=0, f^{-1}\left(N_{1}\right)$ is in $\overline{\mathscr{B}(B)}$ and hence $W=f^{-1}(F)=f^{-1}(G) \cup f^{-1}\left(N_{1}\right)$ is in $\overline{\mathscr{B}(B)}$. Conversely, suppose that $W$ is in ${\overline{\mathscr{B}}(B)^{\nu}}^{\nu}$. Let $\lambda=\nu \circ f^{-1}$. Then $\lambda$ is a Borel probability measure on $R^{n}$. By Lemma $3.1, F$ is $\overline{\mathscr{B}\left(R^{n}\right)^{\lambda}}$-measurable and hence Lebesgue measurable because $\lambda$ is clearly equivalent to Lebesgue measure on $R^{n}$. This completes the proof of (ii).

As an application of Theorem 3.2, we give the following example which shows how to obtain the corresponding result of Skoug's for Yeh-Wiener space [10].

EXAmple 3.3. Let $B=C_{2}[Q], H=C_{2}^{\prime}[Q]$ and $\nu=m_{y}$. Let $a=s_{0}<$ $s_{1}<s_{2}<\cdots<s_{m}=b$ and $c=t_{0}<t_{1}<\cdots<t_{n}=d$ be partitions of $[a, b]$ and $[c, d]$, respectively. Let

$$
h_{i j}(s, t)=\int_{a}^{s} \int_{c}^{t} I_{\left[a, s_{t}\right] \times\left[c, t_{j}\right]}(u, v) d u d v,
$$

for $i=1,2, \ldots, m$ and $j=1,2, \ldots, n$. Then $\left\{h_{i j}\right\}$ is clearly a linearly independent set in $C_{2}^{\prime}[Q]$, and for any $x \in C_{2}[Q]$,

$$
\begin{aligned}
\left(h_{i j}, x\right)^{\sim} & =(\phi) \int_{a}^{b} \int_{c}^{d} D^{2} h_{i j}(s, t) \tilde{d} x(s, t) \\
& =(\phi) \int_{a}^{s_{i}} \int_{c}^{t_{j}} \tilde{d} x(s, t)=x\left(s_{i}, t_{j}\right) .
\end{aligned}
$$

Hence by Theorem 3.2, for any subset $F$ of $R^{m n}$

$$
\begin{aligned}
W & =\left\{x \in C_{2}[Q]:\left(\left(h_{11}, x\right)^{\sim}, \ldots,\left(h_{m n}, x\right)^{\sim}\right) \in F\right\} \\
& =\left\{x \in C_{2}[Q]:\left(x\left(s_{1}, t_{1}\right), \ldots, x\left(s_{m}, t_{n}\right)\right) \in F\right\}
\end{aligned}
$$


is in $\overline{\mathscr{B}\left(C_{2}[Q]\right)^{m}}$ if and only if $F$ is Lebesgue measurable in $R^{m n}$, and furthermore, $W$ is in $\mathscr{B}\left(C_{2}[Q]\right)$ if and only if $F$ is Borel measurable in $R^{m n}$.

Corollary 3.4. Let $(H, B, \nu)$ be an abstract Wiener space, and let $\left\{h_{1}, h_{2}, \ldots, h_{n}\right\}$ be a linearly independent set in $H$. Let $G$ be a complex-valued function defined on $R^{n}$. Then the functional

$$
F(x)=G\left(\left(h_{1}, x\right)^{\sim}, \ldots,\left(h_{n}, x\right)^{\sim}\right)
$$

on $B$ is $\overline{\mathscr{B}(B)}^{\nu}$-measurable $(\mathscr{B}(B)$-measurable) if and only if $G$ is Lebesgue (Borel, resp.) measurable in $R^{n}$.

4. Scale-invariant measurability and translations in abstract Wiener space. Let $(H, B, \nu)$ be an abstract Wiener space. For any $\alpha>0$, let $T_{\alpha}$ : $B \rightarrow B$ be the transformation defined by $T_{\alpha}(x)=\alpha x$ and $\nu_{\alpha}$ the Borel measure on $B$ defined by $\nu \circ T_{\alpha}^{-1}$. Then $\nu_{1}=\nu$ and $\nu_{\alpha}(A)=\nu_{1}\left(\alpha^{-1} A\right)$ for every $A$ in $\mathscr{B}(B)$. Let $\mathscr{S}_{\alpha}=\overline{\mathscr{B}(B)^{\nu}}, \quad \mathscr{S}=\bigcap_{\alpha>0} \mathscr{S}_{\alpha}, \mathscr{N}_{\alpha}=\left\{A \in \mathscr{S}_{\alpha}\right.$ : $\left.\nu_{\alpha}(A)=0\right\}$ and $\mathscr{N}=\bigcap_{\alpha>0} \mathscr{N}_{\alpha}$. Every set in $\mathscr{S}(\mathscr{N})$ is called scale-invariant measurable (scale-invariant null, resp.) set. In this section, we extend the results of [6] on scale-invariant measurable sets, translations and scale-invariant measurable functions in Wiener space to abstract Wiener space.

Now, extensions to the abstract Wiener space setting of the results contained in Propositions 2 to 4 and Theorem 5 of [6] can be shown to hold using exactly the same arguments as given in [6]. For brevity we omit these statements and refer the reader to [6]. Further, the proof of the following two results in the abstract Wiener space setting can also be given using similar techniques as used in [6]. However, for completeness, we include the statements and proof of these results.

PROPOSITION 4.1. For every $\alpha>0, \mathscr{B}(B) \varsubsetneqq \mathscr{S}_{\varsubsetneqq} \mathscr{S}_{\alpha}$.

Proof. The proof of the containments, $\mathscr{B}(B) \subset \mathscr{S} \subset \mathscr{S}_{\alpha}$ is clear from the fact that $\mathscr{B} \subset \mathscr{S}_{\alpha}$ for every $\alpha>0$ and the equaltiy $\mathscr{S}=\bigcap_{\alpha>0} \mathscr{S}_{\alpha}$. The proof of $\mathscr{B}(B) \neq \mathscr{S}$ can be easily shown by using Theorem 3.2. To show that $\mathscr{S} \neq \mathscr{S}_{\alpha}$, let $\alpha, \beta>0$ with $\alpha \neq \beta$. Then by using Theorem 3.2, we can find a subset $W$ of $B$ such that $W$ is not in $\mathscr{S}_{1}$. Clearly $W \cap C_{\alpha}$ is not in $\mathscr{S}_{\alpha}$. Now let $A=\beta^{-1}\left(W \cap C_{\alpha}\right)$. Then $A$ is in $\mathscr{N}_{\alpha}$ and hence $A$ is in $\mathscr{S}_{\alpha}$. But $\beta A=W \cap C_{\alpha}$ is not in $\mathscr{S}_{\alpha}$. Thus $A$ is in $\mathscr{S}_{\alpha}$, but not in $\mathscr{S}_{\beta}$ and so not in $\mathscr{S}$. 
THEOREM 4.2. Let $f$ be any function from $(0, \infty)$ to $[0,1]$. Then there exists $A$ in $\mathscr{S}$ such that $\nu_{1}(\alpha A)=f(\alpha)$ for all $\alpha>0$.

Proof. We first show that for each $\alpha>0$, there exists $A_{\alpha} \subset C_{\alpha}$ such that $A_{\alpha}$ is in $\mathscr{S}_{\alpha}$ and $\nu_{\alpha}\left(A_{\alpha}\right)=f\left(\alpha^{-1}\right)$. To show this, let $f\left(\alpha^{-1}\right)=p$ and $h(\neq 0) \in H$. Then there exists a real number $a_{p}$ such that

$$
\frac{1}{\sqrt{2 \pi|h|}} \int_{-\infty}^{a_{p}} \exp \left\{-\frac{u^{2}}{2|h|}\right\} d u=p
$$

Let $W=\left\{x \in B:(h, x)^{\sim} \leq a_{p}\right\}$. Then $W$ is in $\mathscr{B}(B)$ and hence in $\mathscr{S}_{1}$ and $\nu_{1}(W)=p$. Let $A_{1}=W \cap C_{1}$. Then $A_{1}$ is in $\mathscr{S}_{1}$ and $\nu_{1}\left(A_{1}\right)=p$. Let $A_{\alpha}=\alpha A_{1}$. Then $A_{\alpha}$ is in $\mathscr{S}_{\alpha}, A_{\alpha} \subset C_{\alpha}$ and $\nu_{\alpha}\left(A_{\alpha}\right)=\nu_{\alpha}\left(\alpha A_{1}\right)=$ $\nu_{1}\left(A_{1}\right)=p=f\left(\alpha^{-1}\right)$. Next, we show that the set $A=\bigcup_{\alpha>0} A_{\alpha}$ is the desired set. $A$ is clearly in $\mathscr{S}$ and $\nu_{1}(\alpha A)=\nu_{\alpha^{-1}}(A)=\nu_{\alpha^{-1}}\left(A_{\alpha^{-1}}\right)=f(\alpha)$, completing the proof of the theorem.

Before we study translations in abstract Wiener spaces, we collect a few more definitions and notation, which will be needed in this section. Let $E$ be a real separable Banach space with the topological dual $E^{*}$ of $E$. Let $(\cdot, \cdot)$ denote the natural dual pairing between $E$ and $E^{*}$. For two Borel probability measures $\lambda_{1}$ and $\lambda_{2}$ on $E$, the convolution $\lambda_{1} * \lambda_{2}$ of $\lambda_{1}$ and $\lambda_{2}$ is defined by

$$
\lambda_{1} * \lambda_{2}(G)=\lambda_{1} \times \lambda_{2}(\{(x, y) \in E \times E: x+y \in G\})
$$

for every Borel set $G$ in $E$. The characteristic functional of a Borel probability measure $\lambda$ on $E$ is defined on $E^{*}$ by

$$
\hat{\lambda}(y)=\int_{E} e^{i(x, y)} d \lambda(x), \quad y \in E^{*} .
$$

It is well-known that $\widehat{\lambda_{1} * \lambda_{2}}(\cdot)=\hat{\lambda}_{1}(\cdot) \cdot \hat{\lambda}_{2}(\cdot)$ and that $\lambda_{1}=\lambda_{2}$ if and only if $\hat{\lambda}_{1}(\cdot)=\hat{\lambda}_{2}(\cdot)$.

LEMMA 4.3. Let $p$ and $q$ be positive real numbers.

(i) $\nu_{p} * \nu_{q}=\nu \sqrt{p^{2}+q^{2}}$ on $\mathscr{B}(B), \overline{\mathscr{B}(B)^{\nu_{p}}}{ }^{* \nu_{q}}=\mathscr{S}_{\sqrt{p^{2}+q^{2}}}$.

(ii) If $T:\left(B \times B, \mathscr{B}(B) \times \mathscr{B}(B), \nu_{p} \times \nu_{q}\right) \rightarrow(B, \mathscr{B}(B))$ is the transformation defined by $T(x, y)=x+y$, then for any $A \subset B, T^{-1}(A)$ is in $\overline{\mathscr{S}}_{p} \times \mathscr{S}_{q}^{\nu_{p}{ }^{*} \nu_{q}}$ if and only if $A$ is in $\mathscr{S}_{\sqrt{p^{2}+q^{2}}}$. Further, we have $\left(\nu_{p} \times \nu_{q}\right) \circ T^{-1}$ $=\nu_{p} * \nu_{q}=\nu \sqrt{p^{2}+q^{2}}$ on $\mathscr{S}_{\sqrt{p^{2}+q^{2}}}$.

(iii) If $T_{p} \times T_{q}:\left(B \times B, \mathscr{B}(B) \times \mathscr{B}(B), \nu_{1} \times \nu_{1}\right) \rightarrow(B \times B, \mathscr{B}(B)$ $\times \mathscr{B}(B))$ is the transformation defined by $T_{p} \times T_{q}(x, y)=(p x, q y)$, then for any $A \underset{\mathscr{P}_{p} \times \mathscr{S}_{q} \times \nu_{q}}{\subset} B \times B,\left(T_{p} \times T_{q}\right)^{-1}(A)$ is in $\overline{\mathscr{S}_{1} \times \mathscr{S}_{1}^{\nu_{1}} \times \nu_{1}}$ if and only if $A$ is in

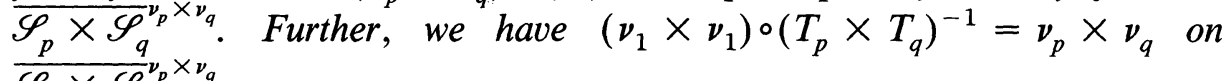
$\frac{p}{\mathscr{S}_{p} \times \mathscr{S}_{q} \nu_{p} \times \nu_{q}}$ 
Proof. (i) Since $\hat{\nu}_{p}(y)=\exp \left\{-\left(p^{2} / 2\right)|y|^{2}\right\}$ for every $y \in B^{*}$ (see [8, p. 78]), it follows that

$$
\widehat{\nu_{p} * \nu_{q}}(y)=\hat{\nu}_{p}(y) \cdot \hat{\nu}_{q}(y)=\exp \left\{-\frac{1}{2}\left(p^{2}+q^{2}\right)|y|^{2}\right\}=\hat{\nu}_{\sqrt{p^{2}+q^{2}}}(y)
$$

for every $y \in B^{*}$. Hence $\nu_{p} * \nu_{q}=\nu \sqrt{p^{2}+q^{2}}$ on $\mathscr{B}(B)$, which implies that $\overline{\mathscr{B}(B)^{\nu_{p}}}{ }^{* \nu_{1}}=\overline{\mathscr{B}(B)} \sqrt{p^{2}+q^{2}}=\mathscr{S}_{\sqrt{p^{2}+q^{2}}}$.

(ii) Suppose $T^{-1}(A)$ is in ${\overline{S_{p} \times \mathscr{S}_{q}}}_{\nu_{p} \times v_{q}}$. Since $T$ is Borel measurable and $\left(\nu_{p} \times \nu_{q}\right) \circ T^{-1}=\nu_{p} * \nu_{q}$ on $\mathscr{B}(B)$, it follows from Lemma 3.1 that $A$

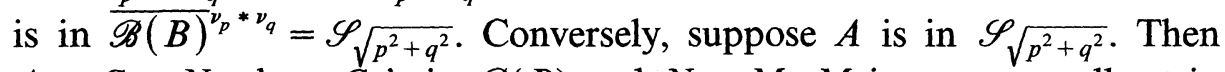
$A=G \cup N$ where $G$ is in $\mathscr{B}(B)$ and $N \subset M, M$ is a $\nu_{p} * \nu_{q}$-null set in $\mathscr{B}(B)$. Thus $\left(\nu_{1} \times \nu_{1}\right) \circ T^{-1}(M)=\nu_{p} * \nu_{q}(M)=\nu \sqrt{p^{2} \times q^{2}}(M)=0$, $T^{-1}(N) \subset T^{-1}(M)$ and $T^{-1}(A)=T^{-1}(G) \cup T^{-1}(N)$. Since $T$ is Borel measurable and $\mathscr{B}(B \times B)=\mathscr{B}(B) \times \mathscr{B}(B), \quad T^{-1}(A)$ is in

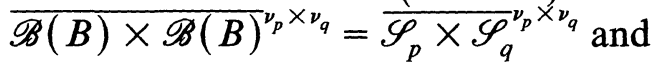

$$
\begin{aligned}
\left(\nu_{p} \times \nu_{q}\right) T^{-1}(A) & =\left(\nu_{p} \times \nu_{q}\right) T^{-1}(G)+\left(\nu_{p} \times \nu_{q}\right) T^{-1}(N) \\
& =\nu_{p} * \nu_{q}(G)+\nu_{p} * \nu_{q}(N)=\nu_{p} * \nu_{q}(A) \\
& =\nu_{\sqrt{p^{2}+q^{2}}}(G)+\nu_{\sqrt{p^{2}+q^{2}}}(N)=\nu_{\sqrt{p^{2}+q^{2}}}(A) .
\end{aligned}
$$

This completes the proof of (ii).

(iii) Let $A$ be a subset of $B \times B$. Since $T_{p} \times T_{q}$ is Borel measurable and $\left(\nu_{1} \times \nu_{1}\right) \circ\left(T_{p} \times T_{q}\right)^{-1}=\nu_{p} \times \nu_{q}$ on $\mathscr{B}(B \times B)=\mathscr{B}(B) \times \mathscr{B}(B)$, it follows from Lemma 3.1 that if $\left(T_{p} \times T_{q}\right)^{-1}(A)$ is in $\overline{\mathscr{S}}_{1} \times \mathscr{S}_{1}{ }^{\nu_{1} \times \nu_{1}}$, then $A$

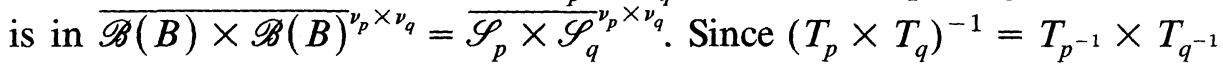
is also a Borel measurable transformation from $(B \times B, \mathscr{B}(B) \times \mathscr{B}(B)$, $\left.\nu_{p} \times \nu_{q}\right)$ to $(B \times B, \mathscr{B}(B) \times \mathscr{B}(B))$ and $\left(\nu_{p} \times \nu_{q}\right) \circ\left(T_{p^{-1}} \times T_{q^{-1}}\right)^{-1}=$ $\nu_{1} \times \nu_{1}$ on $\mathscr{B}(B) \times \mathscr{B}(B)$, by Lemma 3.1 again, it follows that if

$$
\left(T_{p^{-1}} \times T_{q^{-1}}\right)^{-1}\left[\left(T_{p} \times T_{q}\right)^{-1}(A)\right]=A
$$

is in $\overline{\mathscr{S}}_{p} \times \mathscr{S}_{q}{ }_{p} \times v_{q}$, then $\left(T_{p} \times T_{q}\right)^{-1}(A)$ is in $\overline{\mathscr{S}}_{1} \times \mathscr{S}_{1}^{\nu_{1} \times v_{1}}$, proving the first part of (iii). The second part of (iii) can be proved by the similar argument as in the proof of second part of (ii).

The following result follows immediately from Lemma 4.3, (ii) and the Fubini theorem.

Proposition 4.4. Let $p$ and $q$ be positive real numbers. Let $A$ be in $\mathscr{S} \sqrt{p^{2}+q^{2}}$. Then

(i) $A-y$ is in $\mathscr{S}_{p}$ for $\nu_{q}^{-a . s . y}$ and $\nu_{p}(A-y)$ is a $\mathscr{S}_{q}^{- \text {measurable }}$ function of $y$. 
(ii) $A-x$ is in $\mathscr{S}_{q}$ for $\nu_{p}$-a.s.y and $\nu_{q}(A-x)$ is a $\mathscr{S}_{p}$-measurable function of $x$.

(iii)

$$
\begin{aligned}
\nu_{p} * \nu_{q}(A) & =\nu_{p} \times \nu_{q}(\{(x, y): x+y \text { is in } A\}) \\
& =\int_{B} \nu_{p}(A-y) d \nu_{q}(y)=\int_{B} \nu_{q}(A-x) d \nu_{p}(x) .
\end{aligned}
$$

The extensions to the abstract Wiener space setting of the results contained in Corollaries 11 to 15 of [6] now can be easily proved by using Proposition 4.4. For brevity, we omit the statements of these corollaries and their proofs. Now we give a proof of the following result which is the extension of Theorem 9 in [6] to abstract Wiener space. We point out that the present proof is different in spirit from the proof of corresponding result given in [6]: The proof in [6] uses, for instance, a result of Bearman concerning rotations in Wiener space; our proof depends on Lemma 3.1 and on some properties of convolution of measures.

THEOREM 4.5. Let $p$ and $q$ be positive numbers and $f$ a complex-valued function on $B$. Then the following assertions are equivalent:

(a) $f\left(\sqrt{p^{2}+q^{2}} z\right)$ is a $\mathscr{S}_{1}$-measurable function of $z$.

(b) $f(z)$ is a $\mathscr{S}_{\sqrt{p^{2}+q^{2}}}$-measurable function of $z$.

(c) $f(x+y)$ is a $\mathscr{S}_{p} \times \mathscr{S}_{q}$-measurable function of $x$ and $y$.

(d) $f(p x+q y)$ is a $\mathscr{S}_{1} \times \mathscr{S}_{1}$-measurable function of $x$ and $y$.

If any one (and hence all) of (a)-(d) holds, then

$$
\begin{aligned}
\int_{B} f\left(\sqrt{p^{2}+q^{2}} z\right) d \nu_{1}(z) & \stackrel{*}{=} \int_{B} f(z) d \nu \sqrt{p^{2}+q^{2}}(z) \\
& \stackrel{*}{=} \int_{B \times B} f(x+y) d\left(\nu_{p} \times \nu_{q}\right)(x, y) \\
& \stackrel{*}{=} \int_{B \times B} f(p x+q y) d\left(\nu_{1} \times \nu_{1}\right)(x, y)
\end{aligned}
$$

where $\stackrel{*}{=}$ means that the existence of one side implies that of the other with the equality.

Proof. (i) The equivalence of (a) and (b): Define a transformation $T_{\sqrt{p^{2}+q^{2}}}:\left(B, \mathscr{B}(B), \nu_{1}\right) \rightarrow\left(B, \mathscr{B}(B), \nu \sqrt{p^{2}+q^{2}}\right)$ by $T_{\sqrt{p^{2}+q^{2}}}(\mathrm{z})=\sqrt{p^{2}+q^{2}} z$. Then $T \sqrt{p^{2}+q^{2}}$ and $T \sqrt{p^{2}+q^{2}}-1$ are Borel measurable and $\nu_{1} \circ T^{-1} \sqrt{p^{2}+q^{2}}=$ $\nu \sqrt{p^{2}+q^{2}}, \nu \sqrt{p^{2}+q^{2}} \circ T^{-1} \sqrt{p^{2}+q^{2}}-1=\nu_{1}$. Hence by Lemma 3.1, for any Borel set $G$ in the complex plane $C, T_{\sqrt{p^{2}+q^{2}}}^{-1}\left(f^{-1}(G)\right)$ is in $\mathscr{\mathscr { B }}_{(B)^{\nu_{1}}}^{\nu_{1}}=\mathscr{S}_{1}$ if 
and only if $f^{-1}(G)$ is in $\overline{\mathscr{B}(B)} \sqrt[v]{p^{2}+q^{2}}=\mathscr{S}_{\sqrt{p^{2}+q^{2}}}$. Therefore, (a) and (b) are equivalent and the corresponding integration formula follows from the fact that $\nu_{1} \circ T_{\sqrt{p^{2}+q^{2}}}^{-1}=\nu \sqrt{p^{2}+q^{2}}$ on $\mathscr{S}_{\sqrt{p^{2}+q^{2}}}$ and the change of variable theorem ([5], p. 163).

(ii) The equivalence (b) and (c): Let $G$ be a Borel set in $C$ and $T$ the transformation defined in Lemma 4.3, (ii). Then by Lemma 4.3, (ii), $f^{-1}(G)$ is in $\mathscr{S} \sqrt{p^{2}+q^{2}}$ if and only if $T^{-1}\left(f^{-1}(G)\right)=(f \circ T)^{-1}(G)$ is in $\overline{\mathscr{S}}_{p} \times \mathscr{S}_{q}^{\nu_{p} \times \nu_{q}}$. Hence (b) and (c) are equivalent and the corresponding integration formula follows from the fact that $\nu_{p} \times \nu_{q} \circ T^{-1}=\nu \sqrt{p^{2}+q^{2}}$ on $\mathscr{S} \sqrt{p^{2}+q^{2}}$ and the change of variable theorem.

(iii) The equivalence of (c) and (d): Let $G$ be a Borel set in $C$ and $T$ the transformation defined in Lemma 4.3, (ii). Then by Lemma 4.3, (iii), $\left(T_{p} \times T_{q}\right)^{-1}\left(T^{-1}\left(f^{-1}(G)\right)\right)=\left(f \circ T \circ T_{p} \times T_{q}\right)^{-1}(G)$ is in $\overline{\mathscr{S}}_{1} \times \mathscr{S}_{1}{ }^{\nu_{1} \times \nu_{1}}$ if and only if $T^{-1}\left(f^{-1}(G)\right)=(f \circ T)^{-1}(G)$ is in $\overline{\mathscr{S}}_{p} \times \mathscr{S}_{q}^{\nu_{p} \times v_{q}}$. Hence (c) and (d) are equivalent and the corresponding integration formula follows from the fact that $\nu_{1} \times \nu_{1} \circ\left(T_{p} \times T_{q}\right)^{-1}=\nu_{p} \times \nu_{q}$ and the change of variable theorem.

Let $\mathscr{B}(\mathscr{F})\left(\mathscr{S}(\mathscr{F}), \mathscr{S}_{\alpha}(\mathscr{F}), \alpha>0\right)$ denote the class of all complex-valued functions which are defined on a Borel measurable (scale-invariant measurable, $\mathscr{S}_{\alpha}$-measurable, resp.) subset of $B$ and which are measurable with respect to the $\sigma$-algebra $\mathscr{B}(B)\left(\mathscr{S}, \mathscr{S}_{\alpha}\right.$ resp. $)$. The extensions to the abstract Wiener space setting of all the results contained in $\$ 4$ of [6] can be shown to hold by using the same arguments as given in [6]. For brevity, we omit these statements and refer the reader to [6].

Acknowledgments. I would like to thank Professor Balram S. Rajput for his helpful suggestions and assistance in the writing of this paper.

\section{REFERENCES}

[1] R. H. Cameron, The translation pathology of Wiener space, Duke Math. J., 21 (1954), 623-628.

[2] R. H. Cameron and W. T. Martin, The behavior of measure and measurability under change of scale in Wiener space, Bull. Amer. Math. Soc., 53 (1947), 130-137.

[3] K. S. Chang, Scale-invariant measurability in function space, Thesis, University of Nebraska, Lincoln, Neb., 1979.

[4] D. L. Cohn, Measure Theory, Birkhäuser, Boston, 1980.

[5] P. Halmos, Measure Theory, Van Nostrand, New York, 1950.

[6] G. W. Johnson and D. L. Skoug, Scale-invariant measurability in Wiener space, Pacific J. Math., 83 (1979), 157-176.

[7] G. Kallianpur and C. Bromley, Generalized Feynman Integrals Using Analytic Continuation in Several Complex Variables, Stochastic Analysis, M. Pinsky, ed., Marcel-Dekker, 1984. 
[8] H. H. Kuo, Gaussian Measures in Banach Spaces, Lecture Notes in Mathematics, No. 463, Springer, Berlin, 1975.

[9] J. Kuelbs, Abstract Wiener spaces and applications to analysis, Pacific J. Math., 31 (1969), 433-450.

[10] D. L. Skoug, Converses to measurability theorems for Yeh-Wiener space, Proc. Amer. Math. Soc., 57 (1976), 304-310.

[11] , The change of scale and translation pathology in Yeh-Wiener space, Riv. Math. Univ. Parma, 3 (1977), 79-87.

[12] J. Yeh, Stochastic Processes and the Wiener Integral, Marcel Dekker, New York, 1973.

[13] Wiener measure in a space of functions of two variables, Trans. Amer. Math. Soc., 95 (1960), 433-450.

Received April 25, 1986 and in revised form December 2, 1986. This research was supported by the Ministry of Education, Korea (1986).

SOGANG UNIVERSITY

SEOUl 121, Korea 


\section{PACIFIC JOURNAL OF MATHEMATICS EDITORS}

V. S. VARADARAJAN (Managing Editor) University of California Los Angeles, CA 90024 HERBERT ClEMENS University of Utah Salt Lake City, UT 84112

R. FINN

Stanford University

Stanford, CA 94305

\section{HERMANN FLASCHKA} University of Arizona Tucson, AZ 85721

RAMEsh A. GANGolli University of Washington Seattle, WA 98195

VAUghan F. R. JONES

University of California Berkeley, CA 94720

\section{ROBION KIRBY}

University of California

Berkeley, CA 94720

C. C. MOORE

University of California

Berkeley, CA 94720

HAROLD STARK

University of California, San Diego

La Jolla, CA 92093

\section{ASSOCIATE EDITORS}
R. ARENS
E. F. BECKENBACH
B. H. NEUMANN
F. WOLF
K. YOSHIDA (1906-1982)

\section{SUPPORTING INSTITUTIONS}

UNIVERSITY OF ARIZONA

UNIVERSITY OF BRITISH COLUMBIA

CALIFORNIA INSTITUTE OF TECHNOLOGY

UNIVERSITY OF CALIFORNIA

MONTANA STATE UNIVERSITY

UNIVERSITY OF NEVADA, RENO

NEW MEXICO STATE UNIVERSITY

OREGON STATE UNIVERSITY
UNIVERSITY OF OREGON

UNIVERSITY OF SOUTHERN CALIFORNIA

STANFORD UNIVERSITY

UNIVERSITY OF HAWAII

UNIVERSITY OF TOKYO

UNIVERSITY OF UTAH

WASHINGTON STATE UNIVERSITY

UNIVERSITY OF WASHINGTON 


\section{Pacific Journal of Mathematics}

Vol. 130, No. $1 \quad$ September, 1987

K. Adachi, Continuation of bounded holomorphic functions from certain subvarieties to weakly pseudoconvex domains $\ldots \ldots \ldots \ldots \ldots \ldots \ldots$

Erazm Jerzy Behr, Enveloping algebras of Lie superalgebras ........... 9

Dong M. Chung, Scale-invariant measurability in abstract Wiener spaces . . . 27

Peter Gerard Dodds and Bernardus de Pagter, Algebras of unbounded

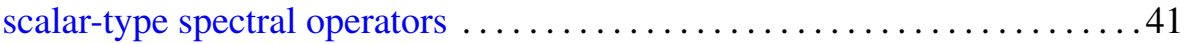

Wu-Yi Hsiang and Hsueh-Ling Huynh, Generalized rotational

hypersurfaces of constant mean curvature in the Euclidean spaces. II . . . .75

Harvey Bayard Keynes and M. Sears, Time changes for $\mathbf{R}^{n}$ flows and suspensions ..................................... 97

Frances Kirwan, Ronnie Lee and Steven Howard Weintraub, Quotients

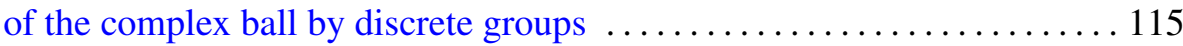

Magnhild Lien, Groups of knots in homology 3-spheres that are not

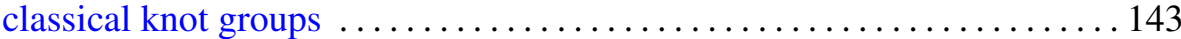

Juan Carlos Migliore, Liaison of a union of skew lines in $\mathbf{P}^{4} \ldots \ldots \ldots \ldots 153$

Jesper M. Møller, Spaces of sections of Eilenberg-Mac Lane fibrations . . . . 171 Daniel Ullman, A generalization of a theorem of Atkinson to noninvariant

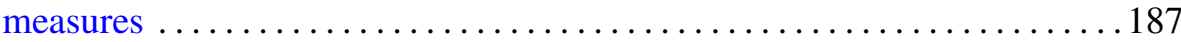

Kohhei Yamaguchi, Operations which detect $\mathscr{P}^{1}$ in odd primary connective

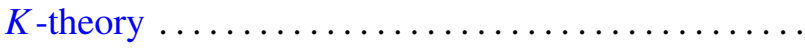

